\title{
SIMULATION OF MAGNETIC RECORDING PROCESS USING A VSM
}

\author{
Yoshiro YONEDA and Junji NUMAZAWA
}

\author{
NHK Science and Technical Research Labs., \\ Kinuta, Setagaya-ku, Tokyo 157, Japan
}

\begin{abstract}
A simulation of the recording process using a VSM (vibrating sample magnetometer) and a media sample with a $6 \mathrm{~mm}$ diameter was used to examine $\mathrm{Co}-\mathrm{Cr}-\mathrm{Ta}$ recording media. The resulting transition was compensated for by calculated true demagnetization fields. The waveform calculated with this transition was more similar to that of actual magnetic recording than the conventional simulation.

Key words: simulation, magnetic recording

\section{INTRODUCTION}

\section{SIMULATION MODEL AND COMPENSATED MAGNETIZATION}

A method of simulating the recording process with a VSM was proposed by Bernards, et al. [1]. In the simulation, demagnetization effects in perpendicular media are fully taken into account, neglecting the decrease of the demagnetization field near a perpendicular transition[2,3]. Simulated reproduced output waveforms by this method are therefore not correct because output waveform strongly depends on the magnetization near a transition.

We compensated for the magnetization transition of a Co-Cr-Ta film simulated in a similar way to the method proposed by Bernards, et al., calculating the demagnetization field. The compensated output waveform was also discussed.

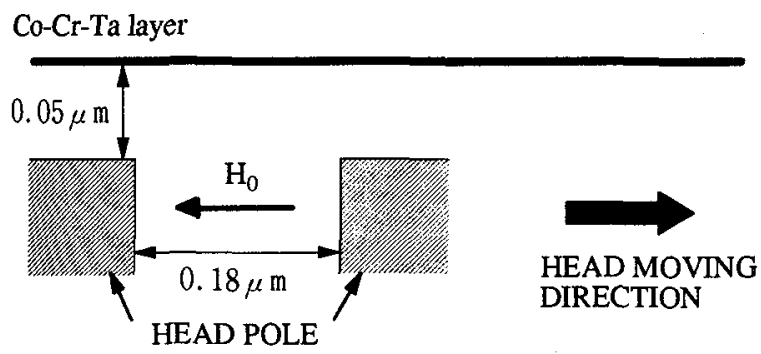

Fig. 1 Head and media model for simulation. $\mathrm{H}_{0}$ is the magnetic field in the gap.

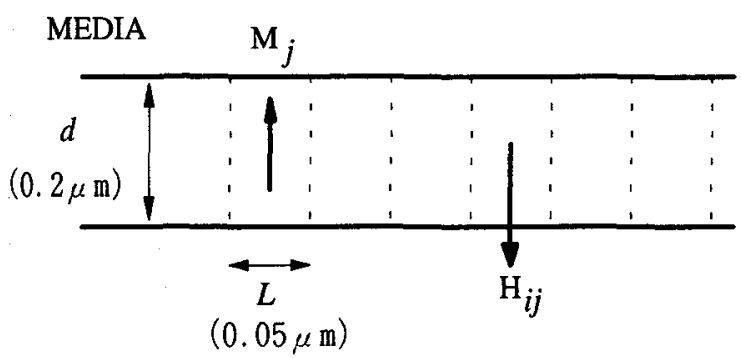

Fig. 2 Media model for calculating the demagnetization field. Subscript $j$ is taken as $i-40$ to $i+40$.
A biaxial VSM was used for the experiments. The sequence of fields sensed by one point in the recording layer when a ring head passed with a stepwise recording current applied to it was calculated in the same way as for [1], and the sequence was applied to a media sample, again in the same way as for [1].

Fig. 1 shows a head and media model and their location for the simulation of the recording process. An infinitely thin film approximation of the recording layer is taken because the field applied by the VSM is homogeneous over the whole sample[2,3].

Fig. 2 shows a media model for calculating true demagnetization fields in the media. The model takes account of the film thickness. The media are divided into several elements. Longitudinal magnetization is neglected in this paper because the Co-Cr-Ta layer we used as a sample has perpendicular anisotropy.

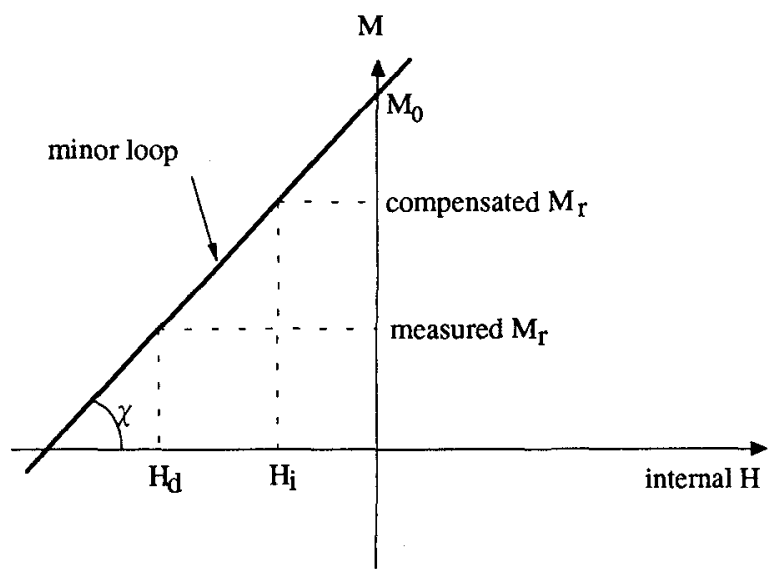

Fig. 3 Line approximation for a minor loop.

$\mathrm{H}_{\mathrm{d}}$ : demagnetization field of the conventional simulation with a VSM $\left(=-4 \pi \mathrm{M}_{\mathrm{r}}\right)$.

$\mathrm{H}_{\mathrm{i}}$ ： true demagnetization field from neighboring points.

$\chi:$ susceptibility. 
The minor loop which the magnetization of one element traced when a head moved away was approximated as a line, as shown in Fig. 3, the equation of which was expressed as

$$
4 \pi \mathrm{M}=4 \pi \mathrm{M}_{0}+\chi \mathrm{H} \quad \text { (in CGS), }
$$

where $\chi$ is the susceptibility at the point.

Since $M=M_{r}$ and $H=-4 \pi M_{r}$ at the end of the recording process,

$$
4 \pi \mathrm{M}_{\mathrm{r}}=4 \pi \mathrm{M}_{0}-\chi 4 \pi \mathrm{M}_{\mathrm{r}}
$$

Consequently, we obtain

$$
4 \pi \mathrm{M}_{0}=4 \pi \mathrm{M}_{\mathrm{r}}(1+\chi) \text {. }
$$

Substituting (3) into (1), we have

$$
4 \pi \mathrm{M}=4 \pi \mathrm{M}_{\mathrm{r}}(1+\chi)+\chi \mathrm{H} \text {. }
$$

We express equation (4) in a matrix form as

$$
4 \pi \mathbf{M}=4 \pi \mathbf{M}_{\mathbf{r}}(1+\chi)+\chi \mathbf{H} \text {. }
$$

The $H$ in equation (4) is the superposition of the fields originating from the magnetization of neighboring elements. The magnetic field $\mathrm{H} i j$ in the center of the $i$ th element from the moment $\mathrm{M} j$ of the $j$ th element is

$$
H_{i j}=-4\left[\tan ^{-1}\left(\frac{(j-i) L+L / 2}{d / 2}\right)-\tan ^{-1}\left(\frac{(j-i) L-L / 2}{d / 2}\right)\right] M j,
$$

where $L$ and $d$ are the length and thickness of an element respectively, as shown in Fig. 2. The range of the subscript $j$ is taken as $i-40$ to $i+40$ in this paper.

We express equation (6) in a matrix form as

$$
\mathbf{H}=-\mathbf{G M} \text {, }
$$

where the element $G_{i j}$ of $\mathbf{G}$ is defined as

$$
\begin{aligned}
G_{i j} & =4\left[\tan ^{-1}\left(\frac{(j-i) L+L / 2}{d / 2}\right)-\tan ^{-1}\left(\frac{(j-i) L-L / 2}{d / 2}\right)\right] \\
& =0 \quad(\mathrm{i}-40 \leqq \mathrm{j} \leqq \mathrm{i}+40) \\
& (\mathrm{j}<\mathrm{i}-40, \mathrm{j}>\mathrm{i}+40) .
\end{aligned}
$$

Substituting (7) into (5), we have

$$
\mathbf{M}=[4 \pi+\chi \mathbf{G}]^{-1} 4 \pi \mathbf{M}_{\mathbf{r}}(1+\chi) \text {. }
$$

The measuring method for $\chi$ using the VSM is now studied. In this paper $\chi$ is assumed to be a scalar constant.

\section{EXPERIMENTAL RESULTS AND DISCUSSION}

\section{A. Simulation without compensation}

Table 1 lists the conditions for the simulation. Fig. 4 shows the M-H loops of a Co-Cr-Ta film with a thickness of $0.2 \mu \mathrm{m}$ used for the experiment. It has an $\mathrm{H}_{c}$ of $1000 \mathrm{Oe}$ and an $\mathrm{MS}_{\mathrm{S}}$ of $460 \mathrm{emu} / \mathrm{cc}$.

Table 1 Conditions for simulation

$\begin{array}{ll}\text { head gap length } & 0.18 \mu \mathrm{m} \\ \text { head media space } & 0.05 \mu \mathrm{m} \\ \text { number of elements } & 40 \\ \text { length of elements } & 0.05 \mu \mathrm{m}\end{array}$

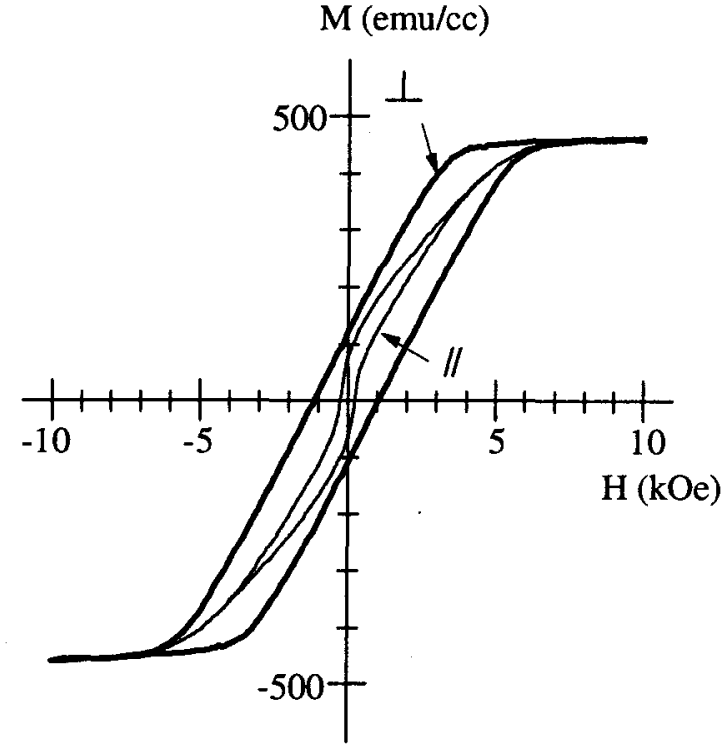

Fig. $4 \mathrm{M}-\mathrm{H}$ loops of the sample.

$\perp:$ perpendicular

$\|$ : in plane

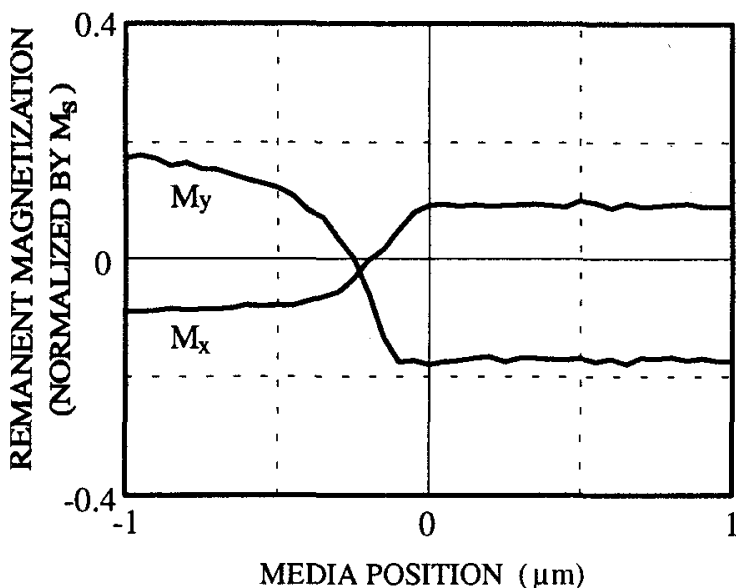

Fig. 5 Simulated transitions of the remanent magnetization.

Fig. 5 shows the remanent vector magnetization transition at an $\mathrm{H}_{0}$ of $6000 \mathrm{Oe} . \mathrm{M}_{\mathbf{x}}$ and $\mathrm{M}_{\mathrm{y}}$ in the figure are the longitudinal and perpendicular components, respectively.

The sample has perpendicular magnetic anisotropy as shown in Fig. 4, but $M_{x}$, as shown in Fig. 5, is relatively large. This is probably caused by the fact that the sample has an initial layer.

The initial layer of magnetic film deposited by sputtering usually has different magnetic properties from the surface under the influence of the substrate. The M-H loop of the in-plane direction shown in Fig. 4 seems a composition of two different $\mathrm{M}-\mathrm{H}$ loops; one is that in the hard axis direction, and the other is that in the easy axis direction, resulting in a jumping loop. This indicates that 
the sample has an initial layer of which the easy axis direction is in plane. The longitudinal component $\mathrm{M}_{\mathrm{x}}$ of the remanent magnetization shown in Fig. 5 probably originates from the initial layer.

Since the magnetization of the initial layer is not considered to contribute to the reproduced output compared with the surface magnetization because of the distance from the head, we calculated the output with the perpendicular component only.

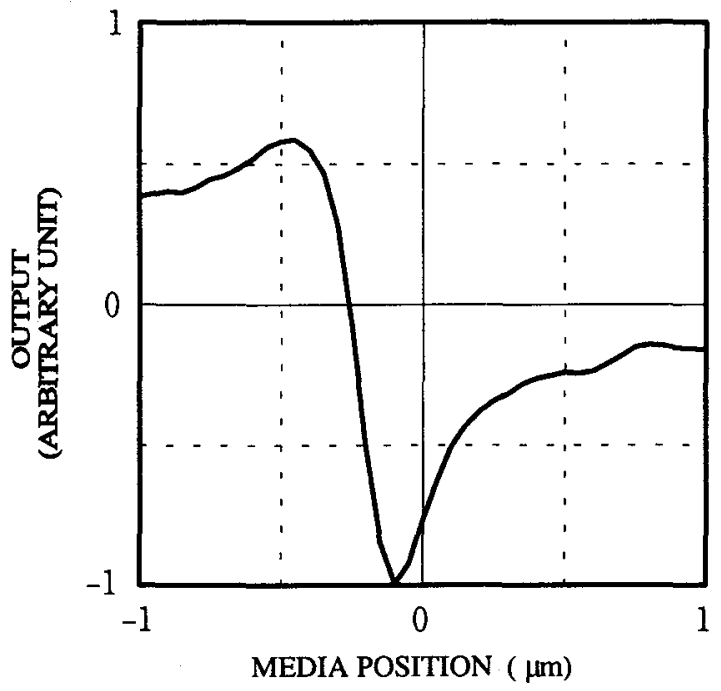

Fig. 6 Simulated reproduced waveform using perpendicular component only.

Fig. 6 shows the simulated reproduced waveform When the output was calculated, it was assumed that the remanent magnetization at points not drawn in Fig. 5 was equal to that at $-1 \mu \mathrm{m}$ or $+1 \mu \mathrm{m}$ in the figure.

For comparison, the actual reproduced output of the film was measured using a drum tester. Table 2 lists the conditions for the experiment. Fig. 7 is a photograph of the reproduced output.

The reproduced waveforms of both the simulation and the actual recording experiment are asymmetric di-pulses, but the slopes of the leading and trailing edges of the dipulse of the actual recording experiment are steeper than those of the simulation.

Table 2 Conditions of recording experiment

\begin{tabular}{cl}
\hline Head: gap length & $0.18 \mu \mathrm{m}$ \\
track width & $20 \mu \mathrm{m}$ \\
number of turns & 30 \\
Velocity & $10.7 \mathrm{~m} / \mathrm{s}$ \\
Signal frequency & $1 \mathrm{MHz}$ \\
(rectangular current) & \\
\hline
\end{tabular}

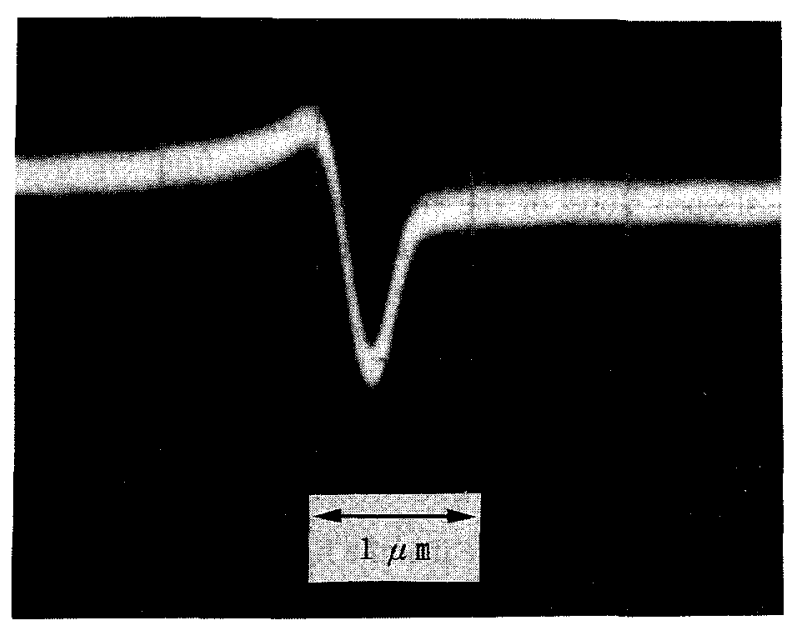

Fig. 7 Photograph of reproduced output.

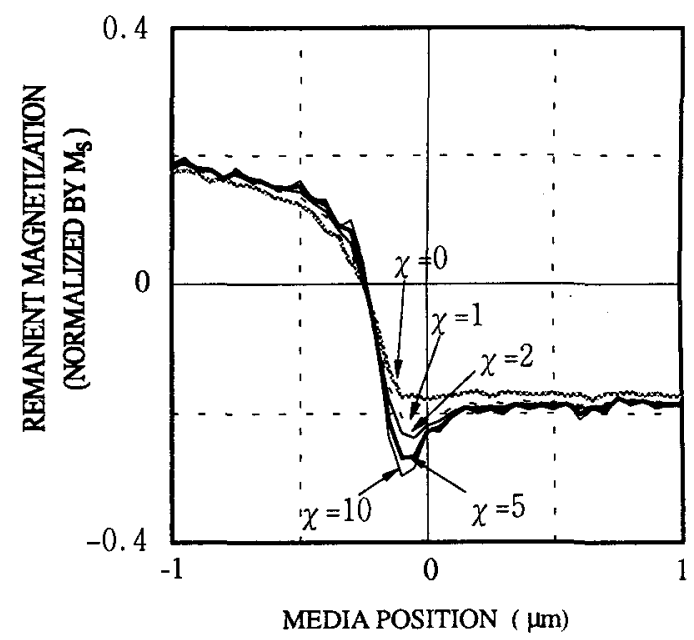

Fig. 8 Compensated transitions of the perpendicular remanent magnetization component with different values of $\chi$. The transition for $\chi=0$ is as same as shown in Fig. 5.

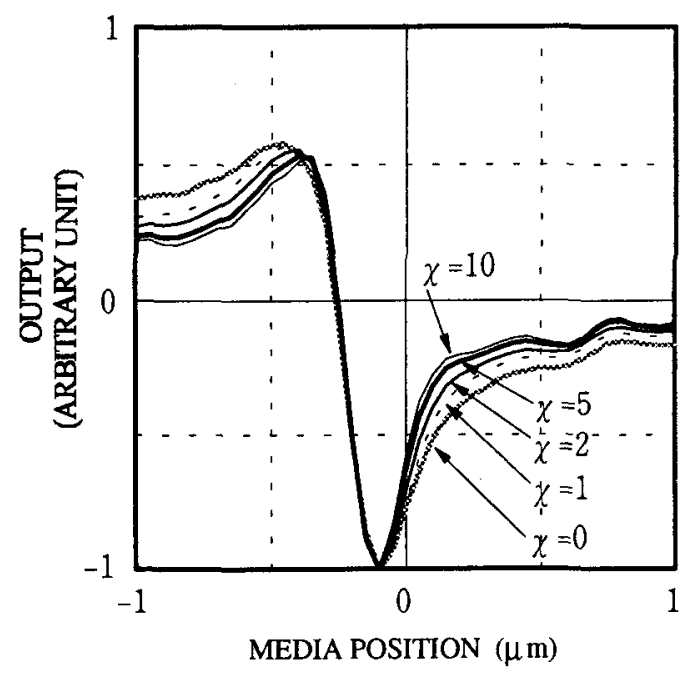

Fig. 9 Compensated waveforms with different values of $\chi$. The waveform for $\chi=0$ is as same as shown in Fig. 6 . 


\section{$B$. Simulation results with compensation}

Fig. 8 shows compensated transitions for different values of $\chi$ as a parameter. The transition for $\chi=0$ is as same as that shown in Fig. 5. We can see from Fig. 8 that the steeper slope of the magnetic reversal point and a deeper undershoot after the magnetic reversal are observed as $\chi$ increases.

Fig. 9 shows the simulated waveforms calculated from the transitions shown in Fig. 8. The slopes of the leading and trailing edges of the di-pulse become steeper as $\chi$ increases, and accordingly the waveforms for a $\chi$ of more than 5 are similar to Fig. 7 .

These figures indicate that the figure of the reproduced waveform in the actual recording experiment can be explained not with the simulation results without compensation but with those with compensation. Since the figure of the waveform influences high density recording characteristics, the simulated high density recording characteristics of both methods will be different. Fig. 10 shows the simulated output dependencies on the linear recording density of both methods. In the simulation the recording current was assumed to be a rectangular wave. A $\chi$ of 5 was applied to the calculation. As expected above, the result with compensation displays better characteristics than that without compensation.

We can conclude from these data that it is very important to compensate for the transition in order to analyze the recording process of media using a VSM. It is essential to measure the susceptibility at each element with a VSM.

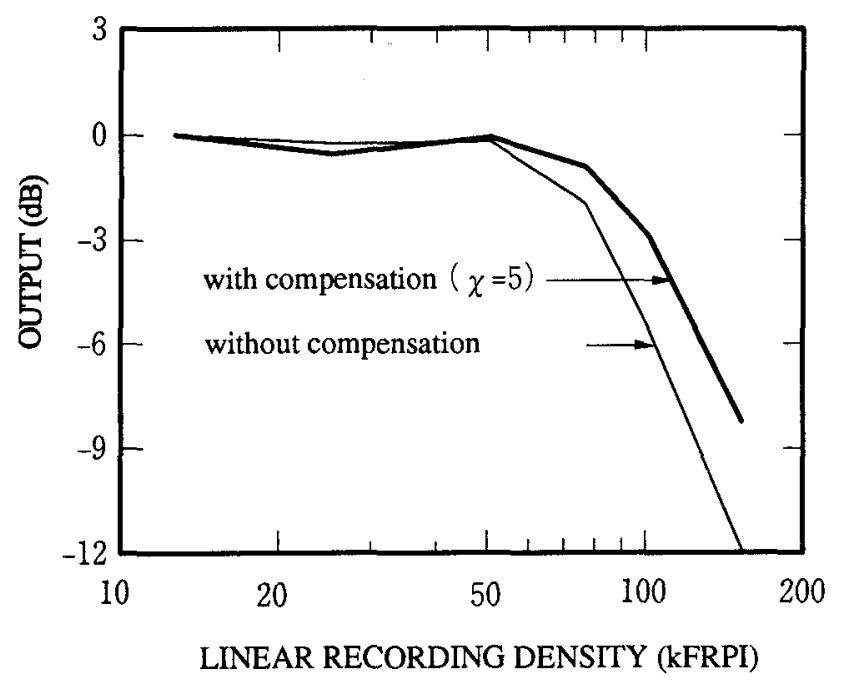

Fig. 10 Simulated high density recording characteristics.

\section{CONCLUSION}

We compensated for the transition of a Co-Cr-Ta film calculated with the conventional simulation of the recording process using a VSM. As a result, the reproduced waveform was more similar to that of actual magnetic recording than the simulation result without compensation.

The remaining issue is to measure the susceptibility of each point of media using the VSM and apply it to equation (8).

\section{ACKNOWLEDGMENT}

The authors would like to thank Mr. Kiyoshi Kuga and Mr. Hideaki Yoshimoto for preparation of the media sample, Mr. Kiyohiko Ishikawa for his cooperation in measuring actual read/write characteristics, and Mr. Hideo Oshima, director of their research division, for his helpful advice on their studies.

\section{REFERENCES}

[1] J.P.C. Bernards, G.J.P. van Engelen, C.P.G. Schrauwen, H.A.J. Cramer, S.B. Luitjens; IEEE Trans. on Magn. 26(1990) p.216

[2] J.P.C. Bernards, G.J.P. van Engelen, C.P.G. Schrauwen, H.A.J. Cramer, S.B. Luitjens; IEEE Trans. on Magn. 26(1990) p.2289

[3] J.P.C. Bernards, G.J.P. van Engelen, C.P.G. Schrauwen, H.A.J. Cramer, S.B. Luitjens; J. Magn. Soc. Japan IS S2(1991) 